\title{
Safety and efficacy of retroperitoneal sutureless zero ischemia laparoscopic partial nephrectomy for low nephrometry score masses
}

\author{
Emanuele Corongiu ${ }^{1}$, Pietro Grande ${ }^{2}$, Angelo Di Santo ${ }^{1}$, Giorgio Pagliarella ${ }^{1}$, Stefano Squillacciotti ${ }^{1}$, \\ Emanuele Liberati ${ }^{1}$, Alessandra Zampelli ${ }^{1}$, Valerio Olivieri ${ }^{3}$, Michele Innocenzi ${ }^{4}$, Flavio Forte ${ }^{1}$ \\ ${ }^{1}$ Department of Urology, M.G. Vannini Hospital, Rome, Italy; \\ 2 Sorbonne Université, Assistance Publique-Hôpitaux de Paris, Pitié Salpétière, Urology Department, Paris, France; \\ ${ }^{3}$ Department of Urology, Ivrea Hospital, ASL TO 4, Ivrea, Italy; \\ ${ }^{4}$ Department of Urology, Bambin Gesù Hospital, Rome, Italy.
}

\begin{abstract}
Summary Objectives: To evaluate oncological feasibility and oncological and functional results of retroperitoneal sutureless zero ischemia laparoscopic partial nephrectomy (LPN).

Patients and methods: Patients with posterior renal masses with low nephrometry score $(R E N A L \leq 7)$ treated who underwent retroperitoneal sutureless zero ischemia.in a single center from January 2016 to November 2017. Clinical, surgical and pathological data were prospectively collected. Complications were reported according to the modified Clavien classification. Results: Retroperitoneal sutureless zero ischemia laparoscopic partial nephrectomy was performed on 15 patients. The indication for nephron-sparing surgery was elective in 11 (73\%) patients and imperative in $4(27 \%)$. Median RENAL score was 5 (IQR: 5-7), median tumor diameter 25 mm (IQR: 20-35). In 11 cases, the tumor was located polar (85\%), and in 2 cases hilar (15\%). There were no intraoperative complications. No cases were converted to radical nephrectomy, and in no case parenchyma suture was necessary. Median operative time was $90 \min$ (IQR:40-150), in no case clamping of the renal artery was necessary, median hospital stay was 4 days, median estimated blood loss (EBL) was 310 (180-500) ml. Pathological analysis showed renal cell carcinoma in 11 patients (85\%), $9(60 \%)$ staged Tla and $2(13 \%)$ Tlb. In 4 (27\%) an oncocytoma was found. There were no positive surgical margins. One patient developed a major postoperative complication (postoperative renal bleeding requiring super-selective embolization). Trifecta rate was $93 \%$.

Conclusions: Sutureless retroperitoneal zero ischemia LPN for the treatment of low-complexity posterior renal masses showed to be safe and feasible. Longer follow-up and higher numbers of patients are, however, warranted to draw definitive conclusions on functional outcomes.
\end{abstract}

KEY WORDS: Laparoscopy; Kidney cancer; Oncological surgery; Partial nephrectomy; Nephron sparing surgery.

Submitted 12 July 2019; Accepted 21 July 2019

\section{INTRODUCTION}

Renal cell carcinoma represents 2-3\% of all cancers, with the highest incidence in Western countries. Despite this, its mortality is slightly decreasing due to earlier detection and improved surgical and non-surgical treatments made available in the last decades (1). Radical nephrectomy (RN) has represented the gold standard approach for years, lately different nephron sparing (NS) approaches (enucleo-resection, wedge resection, pure enucleation etc.) have been proposed to minimize impact on renal function granting optimal oncologic control. Multiple retrospective series have demonstrated a comparable cancer specific survival (CSS) for NS vs. RN for patients with organ-confined RCC of limited size (pT1) (2 4) making this approach the new gold standard for Tl masses. Both EAU and AUA guidelines recognize PN as a valuable option to be performed whenever feasible irrespective of the surgical approach (open or minimally invasive) (1, 5). Both transperitoneal and retroperitoneal approaches have shown to provide similar results in terms of oncologic and surgical safety, with the retroperitoneal one being fitter for posterior located masses (6-8).

Nevertheless, improvements need to be performed to implement post-operative renal function preservation. Both ischemia time and parenchyma loss have been demonstrated to contribute to post-operative renal damage $(9,10)$. Along with complete tumor extirpation without complications, the primary goal for an ideal PN is the maximal preservation of renal function (RF) (11). Indeed, as suggested by extensive evidence, an impaired postoperative RF increases the risk of cardiovascular disease, use of specialized health care and death (12). With this regard, quantity and quality of preserved renal parenchyma are the most important determinants of functional recovery after surgery, with type and duration of ischemia possibly playing a secondary role (10).

Considering this, efforts should be done in both reducing (or eliminating, whenever feasible) ischemia time and reducing the amount of healthy parenchyma removed during tumor resection or destroyed with the haemostatic suture.

Objective of this study is to describe retroperitoneoscopic sutureless zero ischemia partial nephrectomy technique assessing its feasibility and safety, as well as shortterm oncologic and functional outcomes. 


\section{Patients And Methods}

\section{Study population}

From January 2016 to November 2017, 40 consecutive patients with localized renal masses were treated by a single experienced surgeon (FF) in a single institution, with zero ischemia tumor enucleation PN. Every patient gave written informed consent to be included in our prospectively maintained institutional database, where clinicopathological data as well as follow-up and complication data of every patient were recorded. Approval for the study was granted by the hospital ethics committee and the study conformed to the provisions of the Declaration of Helsinki. Comorbidity status was assessed using Charson Comorbidity Index (CCI) (13) and the American Association of Anesthesiologists (ASA) scores (14).

\section{Clinical procedure and patient monitoring}

Fifteen patients with low nephrometry score $($ RENAL $\leq 7$ ) renal masses, located on the posterior surface, were treated using retroperitoneal sutureless zero ischemia approach. Pre-operative exclusion criteria for sutureless technique was tumor close contact with collecting system at preoperative CT scan. The remaining patients treated at the hospital during the study timeframe underwent both transperitoneal PN according to mass location and, for those, sutures were used only for the more complex neoplasms, or to repair damaged collecting systems. Hemoglobin was dosed preoperatively, then 24 hours after intervention to detect potential postoperative bleeding. Renal function was assessed using the estimated glomerular filtration rate (eGFR), calculated with the modification of diet in renal disease (MDRD) equation (15) preoperatively, at discharge and at 3-mo post-operative visit.

\section{Study endpoint}

Primary endpoint was to assess feasibility and safety of retroperitoneal zero ischemia sutureless partial nephrectomy, secondary endpoint was renal function preservation and short-term oncologic outcomes.

\section{Figure 1.}

Flank position, angled at IX-X coastal level for the left side and angled at X-XI coastal level for the right side to better expose the triangle of Petit.



much distance as possible from the peritoneal reflection. The posterior trocar, $2 \mathrm{~cm}$ higher than the optical trocar, on the posterior axillary or in some cases (depending on the body shape) between the angular line of the scapula and the posterior axillary line. As this trocar is placed through the mass of the large dorsal muscle limiting movements in laterality we prefer to use a 5-mm trocar. A $4^{\text {th }}$ trocar, usually placed at the apex of the $12^{\text {th }}$ coast is inserted during the intervention.

An intra-abdominal pressure between 12 and $15 \mathrm{mmHg}$ was used during the entire intervention.

\section{Identification of the mass and resection}

The peritoneal reflection until the vena cava (right side) or ureter (left side) is identified. Subsequently, the surface of the psoas muscle is released, under the Gerota capsule, up to its proximal insertion on the diaphragm (Figure 2a). Laterally, the lateral-conal fascia is freed until the diaphragmatic insertion, to achieve posterior and lateral mobilization of the pre-renal fat. Gerota's fascia is bluntly dissected cranially, along the renal convexity, starting from its lower apex. The dissection of the perinephric fat is continued until the mass is identified (Figure 2b).

\section{Surgical technique}

Patient's position and access

to the retroperitoneum

The patient was placed on flank position, angled at IX-X coastal level for the left side and angled at X-XI coastal level for the right side to better expose the triangle of Petit (Figure 1).

The first incision was performed $3 \mathrm{~cm}$ above the iliac ridge, on the mid-auxiliary line. A blunt dissection of the anterior component of the lumbar-dorsal fascia with Mayo scissors and subsequently digital dissection is performed until the identification of the postero-lateral surface of the psoas muscle and the distal portion of Gerota's fascia. An inflatable space maker balloon is used to develop the retroperitoneal space. The remaining 2 trocars are placed under digital guide: the anterior one $(10 \mathrm{~mm}) 2$ $\mathrm{cm}$ above the optical trocar, keeping as

\section{Figure 2.}

A) surface of the psoas muscle is released; $\boldsymbol{B})$ dissection of the perinephric fat until the mass is identified; $\mathbf{C}$ ) identifying the vascular pole of the neoplasm; $\boldsymbol{D})$ coagulation with bipolar forceps along the enucleation margin.

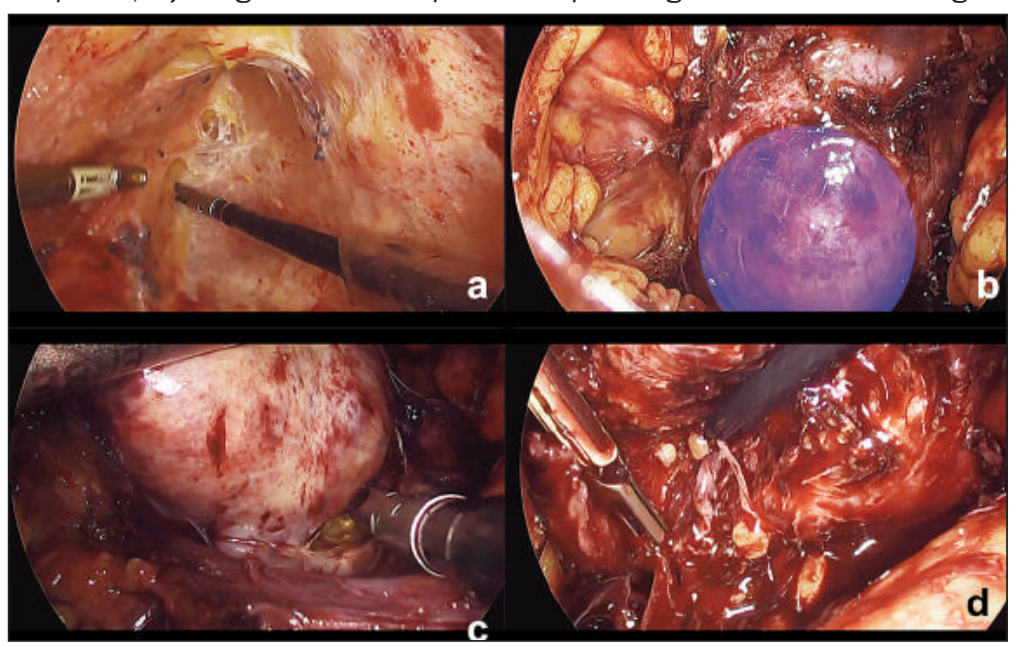


Figure 3.

Fibrin-based glue is applied on the resection area.

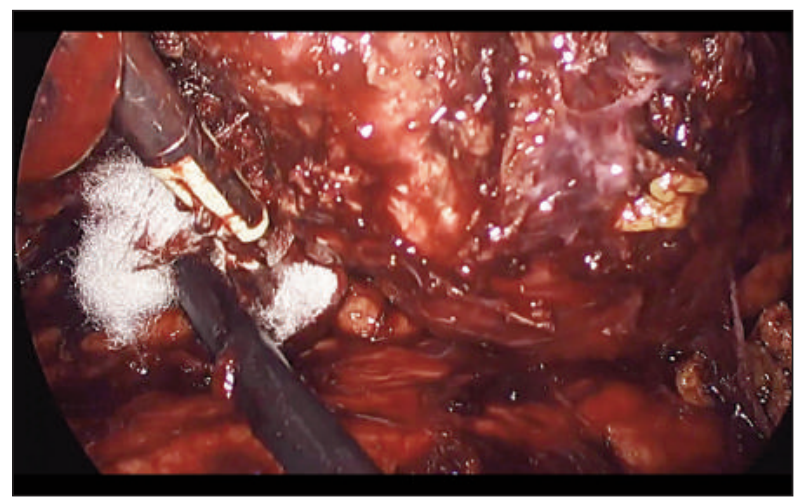

Identification of the vascular supply and hemostasis

The resection of the mass is performed with the aim of tumor enucleation whenever possible, using monopolar scissors and suction. This step is performed totally clampless, for this reason, identifying as soon as possible the vascular pole of the neoplasm, who's generally represented by an arterial branch lying on the resection burden, is crucial. Useful for this purpose is the blunt dissection performed with the tip of the suction device or with the bipolar forceps (Figure 2c), rising the lesion bottom to top, while the scissor proceeds with mechanical detachment, pandering to the convexity of the lesion. The vascular pole of the renal tumor is usually coagulated with bipolar forceps, except for some cases where the use of a titanium clip is mandatory due to the presence of larger vessels. The margins of the enucleation are not particularly bloody when the neoplasm is located at the lower pole, on the convexity (along the Brodel line, at whose level the interlobar vessels are thinner and fold medially towards the renal sinus), or at the upper pole. In these cases, coagulation with bipolar forceps is sufficient along the enucleation margin (Figure 2d). Conversely, when lesions are located on the posterior surface of the kidney, the risk to have more than one vascular pole is higher. In those cases, clipping of larger arterial branches could be necessary.

\section{Final remarks}

At the end of the procedure the pneumoretroperitoneum is lowered until 5 $\mathrm{mm} \mathrm{Hg}$ to check for any residual bleeding.

Thereafter, fibrin-based glue (Floseal Baxter, USA) or gauze (Tabotamp Ethicon, Inc., Somerville, NJ) is applied on the resection area (Figure 3 ). In our experience this practice resulted more useful for lesions on the posterior surface of the kidney, where intra-renal vascular branches are more extensive.
Surgical specimen is removed using an endo-bag through the anterior trocar: this to avoid further stress, at the margins of the lumbar-dorsal fascia, which would lead to greater risk of hernial failure. No drain is left in place.

\section{Postoperative measures}

Pathological examination was performed by a dedicated uro-pathologist according to the 2016 World Health Organization criteria (16) and to the Fuhrman classification (17). Positive surgical margin was defined as cancer cells at the level of the inked parenchymal excision surface. The severity of complications was reported according to the modified Clavien Classification (18).

The Trifecta rate was calculated as the combination of warm ischemia time $<25 \mathrm{~min}$, negative surgical margins and no complications (up to 90-day follow-up)(11). Patients were followed in outpatient setting 3 months after surgery and every 6 months for the first two years, then yearly. The follow-up protocol included: clinical visit, physical examination and a metabolic panel at 1,3 and 6 month, annually thereafter; computed tomography (CT) at 6 and 12 month, annually thereafter. Patients were planned to be discharged at $4^{\text {th }}$ post-operative day as per hospital protocol, eGFR was assessed at discharge and at 3 months post-operative visit.

\section{RESULTS}

The indication for nephron-sparing surgery was elective
Table 1.

Preoperative characteristics.

\begin{tabular}{|c|c|c|c|c|}
\hline & $\begin{array}{l}\text { Sutureless } \\
\text { retroperitoneal }(n=15)\end{array}$ & $\begin{array}{l}\text { Transperitoneal } \\
\quad(n=25)\end{array}$ & $\begin{array}{l}\text { Overall } \\
(n=40)\end{array}$ & $\begin{array}{c}P \\
\text { value }\end{array}$ \\
\hline Age, years, mean (SD) & $64.0(13.70)$ & 61.4 (11.71) & $62.0(10.94)$ & $>0.05$ \\
\hline $\mathrm{BMI}, \mathrm{Kg} / \mathrm{m}^{2}$, mean $(\mathrm{SD})$ & $25.03(2.11)$ & $25.9(2.14)$ & $26.00(2.12)$ & $>0.05$ \\
\hline Sex $m / f$ & $8 / 7$ & $17 / 8$ & $25 / 15$ & $>0.05$ \\
\hline ASA score, n (\%) & & & & $>0.05$ \\
\hline 1 & $3(20)$ & $7(28)$ & $10(25)$ & \\
\hline 2 & $11(73)$ & $16(64)$ & $27(67.5)$ & \\
\hline 3 & $1(7)$ & $2(8)$ & $3(7.5)$ & \\
\hline CCI, median (IQR) & $4(3-4)$ & $4(3-4)$ & $4(3-4)$ & $>0.05$ \\
\hline Affected kidney & & & & $>0.05$ \\
\hline Right & $5(33)$ & $14(56)$ & $19(47.5)$ & \\
\hline Left & $10(67)$ & $11(44)$ & $21(52.5)$ & \\
\hline Tumor size, mm, median (IQR) & $25(20-35)$ & $28(2.5-4)$ & $30(25-35)$ & $>0.05$ \\
\hline RENAL Score & & & & $>0.05$ \\
\hline$<5$ & $3(20)$ & $2(8)$ & $5(12.5)$ & \\
\hline $5-6$ & $8(53)$ & $11(44)$ & $19(47.5)$ & \\
\hline 7 & $4(27)$ & $6(24)$ & $10(25)$ & \\
\hline$>7$ & - & $6(24)$ & $6(15)$ & \\
\hline Tumor location & & & & $>0.05$ \\
\hline Polar & $13(87)$ & $18(72)$ & $31(77.5)$ & \\
\hline Hilar & $2(13)$ & $7(28)$ & $9(22.5)$ & \\
\hline Exophitic rate & & & & $>0.05$ \\
\hline$<50 \%$ & $5(33)$ & $7(28)$ & $12(30)$ & \\
\hline$\geq 50 \%$ & $10(67)$ & $18(72)$ & $28(70)$ & \\
\hline PN indication & & & & $>0.05$ \\
\hline Imperative & $4(27)$ & $2(8)$ & $6(15)$ & \\
\hline Elective & $11(73)$ & $23(92)$ & $34(85)$ & \\
\hline
\end{tabular}




\begin{tabular}{|c|c|c|c|c|}
\hline & $\begin{array}{l}\text { Sutureless } \\
\text { etroperitoneal }(n=15)\end{array}$ & $\begin{array}{c}\text { Transperitoneal } \\
\quad(n=25)\end{array}$ & $\begin{array}{l}\text { Overall } \\
(n=40)\end{array}$ & $\begin{array}{c}P \\
\text { value }\end{array}$ \\
\hline Operative time, min, mean (SD) & $100(31.5)$ & $130(31.4)$ & $115(33.2)$ & $>0.05$ \\
\hline Length of stay, $d$, mean (SD) & $4.13(0.37)$ & $4.7(0.88)$ & $4.55(1.45)$ & $>0.05$ \\
\hline Estimated blood loss, ml, mean (SD & SD) 370 (299) & $425(285)$ & $410(275)$ & $>0.05$ \\
\hline Histology, $n(\%)$ & & & & $>0.05$ \\
\hline Oncocytoma & $4(26.6)$ & $2(8)$ & $6(15)$ & \\
\hline Renal Cell carcinoma & $11(73.4)$ & $23(92)$ & $34(85)$ & \\
\hline Positive surgical margin, $n(\%)$ & $0(0)$ & 0 & $0(0)$ & \\
\hline $\begin{array}{l}\text { Maximal tumour-kidney margin, } \\
\mathrm{mm} \text {, mean (SD) }\end{array}$ & $4.1(0.7)$ & $4.6(1.2)$ & $4.4(0.8)$ & $>0.05$ \\
\hline pT Stage & & & & $>0.05$ \\
\hline $\mathrm{T} 1 \mathrm{a}$ & $9(60)$ & $18(72)$ & $27(67.5)$ & \\
\hline $\mathrm{T} 1 \mathrm{~b}$ & $2(13.3)$ & $3(12)$ & $5(12.5)$ & \\
\hline$\geq \mathrm{T} 2$ & $0(0)$ & $2(8)$ & $2(5)$ & \\
\hline Fuhrman grade (for RCC), $\mathrm{n}(\%)$ & & & & $>0.05$ \\
\hline 1 & $2(13.3)$ & $3(12)$ & $5(12.5)$ & \\
\hline$\|$ & $7(46.7)$ & $18(72)$ & $25(62.5)$ & \\
\hline$\geq|I|$ & $2(13.3)$ & $2(8)$ & $4(10)$ & \\
\hline Complications* & & & & $>0.05$ \\
\hline 1 & $3(20)$ & $2(8)$ & $5(12.5)$ & \\
\hline$\|$ & - & $1(4)$ & $1(2.5)$ & \\
\hline$\geq \mathrm{III}$ & $1(6.7)$ & $1(4)$ & $2(5)$ & \\
\hline Mean (SD) eGFR & & & & $>0.05$ \\
\hline Preoperative & $85.23(29.95)$ & $81.34(24.22)$ & $80.59(24.28)$ & \\
\hline Discharge & $74.77(23.22)$ & $72.34(21.24)$ & $71.89(21.31)$ & \\
\hline 3-Mo post-OP & $81.78(16.77)$ & $79.12(17.10)$ & $69.25(16.52)$ & \\
\hline \multicolumn{5}{|l|}{${ }^{\star}$ According to Clavien-Dindo classification. } \\
\hline & Present study & Simone et al. & Minervini et al. & \\
\hline Overall study population, $n$ & 15 & 380 & 100 & \\
\hline Overall sutureless procedures, $\mathrm{n}(\%)$ & (\%) $15(100)$ & 101(26.6) & $32(32)$ & \\
\hline Transeritoneal & - & $101(26.6)$ & N.A. & \\
\hline Retroperitoneal & $15(100)$ & - & N.A. & \\
\hline Clampless Procedures, $\mathrm{n}(\%)$ & $15(100)$ & $101(100)$ & $18(56.3)$ & \\
\hline \multicolumn{5}{|l|}{ RENAL score } \\
\hline$<5$ & $3(20)$ & $94(93.1)$ & N.A. & \\
\hline $5-6$ & $8(53)$ & $7(6.9)$ & N.A. & \\
\hline 7 & $4(27)$ & - & N.A. & \\
\hline Tumour size, mm, median (IQR) & $25(20-35)$ & $24(15-40)$ & $19(15-21)$ & \\
\hline \multicolumn{5}{|l|}{ Exophytic rate, $n(\%)$} \\
\hline$<50 \%$ & $5(33)$ & N.A. & $5(17)$ & \\
\hline$\geq 50 \%$ & $10(67)$ & N.A. & $27(83)$ & \\
\hline Sex $m / f$ & $8 / 7$ & $63 / 38$ & N.A. & \\
\hline \multicolumn{5}{|l|}{ ASA score } \\
\hline 1 & $3(20)$ & $45(44.6)$ & N.A. & \\
\hline 2 & $11(73)$ & $30(29.7)$ & N.A. & \\
\hline 3 & $1(7)$ & $26(25.7)$ & N.A. & \\
\hline Age, years, median (range) & $68(28-70)$ & $59(45-73)$ & $62(46-80)$ & \\
\hline $\mathrm{BMI}, \mathrm{Kg} / \mathrm{m}^{2}$, meadian (IQR) & $25.0(24.2-27.6)$ & N.A. & $26(19.5-35)$ & \\
\hline \multicolumn{5}{|l|}{ Tumour location } \\
\hline Polar & $13(87)$ & $96(95.1)$ & $32(100)$ & \\
\hline Hilar & $2(13)$ & $5(4.9)$ & - & \\
\hline Operative time, min, median (range) & se) $90(40-150)$ & $60(45-160)$ & $115(80-180)$ & \\
\hline \multicolumn{5}{|l|}{ sCr, mg/dL, median (range) } \\
\hline Preoperative & $0.8(0.6-1.7)$ & $0.9(0.6-1.3)$ & N.A. & \\
\hline At discharge & $0.9(0.6-1.8)$ & - & N.A. & \\
\hline 3-mo post-op & $0.9(0.6-1.9)$ & $1(0.6-1.4)$ & N.A. & \\
\hline \multicolumn{5}{|l|}{ eGFR, ml/min, median (range) } \\
\hline Preoperative & $88(40-135)$ & $96(60-120)$ & N.A. & \\
\hline At discharge & $79(37-124)$ & - & N.A. & \\
\hline 3-mo post-op & $90(36-121)$ & $93(58-125)$ & N.A. & \\
\hline Completed sutureless & $15(100)$ & $97(96)$ & $32(100) \S$ & \\
\hline
\end{tabular}

Table 2.

Post-operative outcomes.

in $11(73 \%)$ patients and imperative in 4 (27\%), of those 3 presented a solitary kidney and one had impaired renal function. Preoperative median (IQR) eGFR was $88(40-135) \mathrm{ml} / \mathrm{min} / 1.73 \mathrm{~m}^{2}$. Median RENAL score was 5 (IQR: 5-7), median tumor diameter $25 \mathrm{~mm}$ (IQR: 20-35); 4 (27\%) patients presented a mass with a RENAL score of 7 . The tumor was located polar in $13(87 \%)$ cases and hilar in 2 (13\%) cases. Further patients' characteristics for the study population compared to the entire cohort of treated patients are listed in Table 1

All interventions were successfully completed, with no report of intraoperative complication. No vascular lesions occurred during the procedure; no cases were converted to radical nephrectomy, in no case the use of parenchymal suture was necessary.

Median (IQR) operative time was 90 (40150) $\mathrm{min}$. All procedures were completed with zero ischemia technique as in no case clamping of the renal artery was necessary. Mean (SD) hospital stay was 4.1 (0.4) days. Median (IQR) GFR was 79 (37-124) at discharge and 90 (36121) at 3-month post-operative visit. Median IQR GRF median (IQR) EBL was 310 (180-500) ml. Pathological analysis showed renal cell carcinoma in 11 patients (73\%), 9 (60\%) staged Tla and 2 (13\%) Tlb. In 4 (27\%) an oncocytoma was found. There were no positive surgical margins. One patient (7\%) developed a major postoperative complication, post-operative renal bleeding requiring super-selective embolization, while 3 (20\%) developed minor complications (Clavien I). Trifecta was achieved in 93\% of the patients treated with sutureless retroperitoneal approach. Furthermore, oncologic and functional results were similar when compared to the transperitoneal cohort (Table 2).

\section{Comment}

The reports on lower incidence of postoperative acute kidney injury and chronic kidney disease (CKD) after off-clamp $\mathrm{PN}$ in the solitary kidney model led to an increased use of this approach for all patients, possibly to avoid the detrimental effect of ischemia on RF (19).

Whereas the functional benefit of the off- 
clamp technique has been suggested by several authors both for patients with solitary and normal contralateral kidney (20-23), most of the studies do not include data on resection and reconstruction technique thus potentially either underestimate or overrate the actual effect of arterial clamping.

Pure tumor enucleation, for those reasons, could help in preserving the maximal amount of healthy parenchyma granting comparable oncologic and surgical outcomes of conventional partial nephrectomy in $\mathrm{T} 1$ renal cancer, as previously reported (24), facilitating a better recovery of post-operative renal function (25), although no general consensus exists yet.

Clampless approach, avoids on one side ischemia-related renal damage $(26,27)$ and on the other side, it permits to avoid any possible vascular damage related to hilum dissection reducing the time of the intervention. Additionally, in experienced hands, clampless approach could facilitate the surgeon to identify major arterial branches and seal it during enucleation.

Renal function was assessed at discharge and at 3months post-op visit as per-hospital protocol, after this period, as previously described, no intervention related variations in RF should occur (28).

Renal function was not particularly affected as reported in Table 2, no de novo CKD grade $\geq 3$ a occurred. Additionally, the only two patients with a GFR in the range of CKD stage 3 did not experience any notable decrease in RF. The transitory decrease in RF evidenced at discharge, and the complete restoration of the original GFR values for most of the patients in our series could be considered a consequence of the off-clamp approach, as well as the enucleative technique.

Previous experiences with sutureless PN were reported, showing that under certain conditions it is a safe and effective procedure $(29,30)$ (Table 3$)$. This study confirms previous experiences, demonstrating that this technique may also be applicable for masses with a RENAL score up to 7 , without compromising oncologic and functional outcomes.

Main limitations of this study include small sample size and retrospective nature of the analysis, although data collection was prospective. Nonetheless, present study is unique as it represents the first, to our knowledge, description of this technique. Furthermore, our series investigates feasibility and safety retroperitoneoscopic clampless sutureless partial nephrectomy, reporting functional outcomes and trifecta rate with a minimum follow-up length of 6 mo.

Further studies, employing renal scintigraphy may help determine the real impact of the technique on the operated kidney parenchyma especially in case of imperative indication.

Although not comparable with larger series of PN, due to the very selective nature of the cohort and the short follow up period, our preliminary experience showed feasibility and safety of sutureless retroperitoneal zero ischemia LPN for the treatment of low-complexity (up to RENAL 7 score) posterior renal masses.

Longer follow-up and higher numbers of patients are, however, warranted to draw definitive conclusions.

\section{References}

1. Ljungberg B, Albiges L, Bensalah K, et al. EAU guidelines on Renal Cell Carcinoma [Internet]. Uroweb. 2018 [cited 2018 Mar 27]. Available from: https://uroweb.org/guideline/renal-cell-carcinoma/

2. Van Poppel H, Da Pozzo L, Albrecht W, et al. A prospective, randomised EORTC intergroup phase 3 study comparing the oncologic outcome of elective nephron-sparing surgery and radical nephrectomy for low-stage renal cell carcinoma. Eur Urol. 2011; 59:543-52.

3. Marchioni M, Preisser F, Bandini M, et al. Comparison of partial versus radical nephrectomy effect on other-cause mortality, cancerspecific mortality, and 30-day mortality in patients older than 75 years. Eur Urol Focus. 2018 Feb 2.

4. Kunath F, Schmidt S, Krabbe L-M, et al. Partial nephrectomy versus radical nephrectomy for clinical localised renal masses. Cochrane Database Syst Rev. 2017; 5:CD012045.

5. Campbell S, Uzzo RG, Allaf ME, et al. Renal Mass and Localized Renal Cancer: AUA Guideline. J Urol. 2017; 198:520-9.

6. Ren T, Liu Y, Zhao X, et al. Transperitoneal approach versus retroperitoneal approach: a meta-analysis of laparoscopic partial nephrectomy for renal cell carcinoma. PloS One. 2014; 9:e91978.

7. Choo SH, Lee SY, Sung HH, et al. Transperitoneal versus retroperitoneal robotic partial nephrectomy: matched-pair comparisons by nephrometry scores. World J Urol. 2014; 32:1523-9.

8. Stroup SP, Hamilton ZA, Marshall MT, et al. Comparison of retroperitoneal and transperitoneal robotic partial nephrectomy for Pentafecta perioperative and renal functional outcomes. World J Urol. 2017; 35:1721-8.

9. Volpe A, Blute ML, Ficarra $V$, et al. Renal ischemia and function after partial nephrectomy: a collaborative review of the literature. Eur Urol. 2015; 68:61-74.

10. Zabell JR, Wu J, Suk-Ouichai C, Campbell SC. Renal ischemia and functional outcomes following partial nephrectomy. Urol Clin North Am. 2017; 44:243-55.

11. Hung AJ, Cai J, Simmons MN, Gill IS. "Trifecta" in partial nephrectomy. J Urol. 2013; 189:36-42.

12. Go AS, Chertow GM, Fan D, et al. Chronic kidney disease and the risks of death, cardiovascular events, and hospitalization. N Engl J Med. 2004; 351:1296-305.

13. Charlson ME, Pompei P, Ales KL, MacKenzie CR. A new method of classifying prognostic comorbidity in longitudinal studies: development and validation. J Chronic Dis. 1987; 40:373-83.

14. Doyle DJ, Garmon EH. American Society of Anesthesiologists Classification (ASA Class). In: StatPearls [Internet]. Treasure Island (FL): StatPearls Publishing; 2018 [cited 2018 Jun 4]. Available from: http://www.ncbi.nlm.nih.gov/books/NBK441940/

15. Levey AS, Bosch JP, Lewis JB, et al. A more accurate method to estimate glomerular filtration rate from serum creatinine: a new prediction equation. Modification of Diet in Renal Disease Study Group. Ann Intern Med. 1999; 130:461-70.

16. Moch H, Cubilla AL, Humphrey PA, et al. The 2016 WHO classification of tumours of the urinary system and male genital organs-Part A: renal, penile, and testicular tumours. Eur Urol. 2016; 70:93-105.

17. Fuhrman SA, Lasky LC, Limas C. Prognostic significance of morphologic parameters in renal cell carcinoma. Am J Surg Pathol. 1982; 6:655-63.

18. Dindo D, Demartines N, Clavien P-A. Classification of surgical complications: a new proposal with evaluation in a cohort of 6336 patients and results of a survey. Ann Surg. 2004; 240:205-13. 
19. Mir MC, Ercole C, Takagi T, et al. Decline in renal function after partial nephrectomy: etiology and prevention. J Urol. 2015; 193:1889-98

20. Lane BR, Russo P, Uzzo RG, et al. Comparison of cold and warm ischemia during partial nephrectomy in 660 solitary kidneys reveals predominant role of nonmodifiable factors in determining ultimate renal function. J Urol. 2011; 185:421-7.

21. Thompson RH, Lane BR, Lohse CM, et al. Every minute counts when the renal hilum is clamped during partial nephrectomy. Eur Urol. 2010; 58:340-5.

22. Kim EH, Tanagho YS, Sandhu GS, et al. Off-clamp robot-assisted partial nephrectomy for complex renal tumors. J Endourol. 2012; 26:1177-82.

23. Kaczmarek BF, Tanagho YS, Hillyer SP, et al. Off-clamp robotassisted partial nephrectomy preserves renal function: A multi-institutional propensity score analysis. Eur Urol. 2013; 64:988-93.

24. Cao D-H, Liu L-R, Fang Y, et al. Simple tumor enucleation may not decrease oncologic outcomes for $T 1$ renal cell carcinoma: A systematic review and meta-analysis. Urol Oncol. 2017; 35:661.e15661.e21.
25. Dong W, Gupta GN, Blackwell RH, et al. Functional comparison of renal tumor enucleation versus standard partial nephrectomy. Eur Urol Focus. 2017; 3:437-43.

26. Porpiglia F, Bertolo R, Amparore D, et al. Evaluation of functional outcomes after laparoscopic partial nephrectomy using renal scintigraphy: clamped vs clampless technique. BJU Int. 2015; 115:606-12.

27. Simone G, Gill IS, Mottrie A, et al. Indications, techniques, outcomes, and limitations for minimally ischemic and off-clamp partial nephrectomy: a systematic review of the literature. Eur Urol. 2015; 68:632-40.

28. Porpiglia F, Fiori C, Bertolo R, et al. Long-term functional evaluation of the treated kidney in a prospective series of patients who underwent laparoscopic partial nephrectomy for small renal tumors. Eur Urol. 2012; 62:130-5.

29. Simone G, Papalia R, Guaglianone S, Gallucci M. "Zero ischaemia", sutureless laparoscopic partial nephrectomy for renal tumours with a low nephrometry score. BJU Int. 2012; 110:124-30.

30. Minervini A, Siena G, Tuccio A, et al. Sutureless hemostatic control during laparoscopic NSS for the treatment of small renal masses. Surg Innov. 2014; 21:32-8.

\section{Correspondence}

Emanuele Corongiu, MD (Corresponding Author)

emanuele.corongiu@libero.it

Angelo Di Santo

adisanto1978@gmail.com

Giorgio Pagliarella

giorgiopagliarella@hotmail.com

Stefano Squillacciotti

stefano.squillacciotti@gmail.com

Emanuele Liberati

emanuele.liberati@libero.it

Alessandra Zampelli

a.zampelli@libero.it

Flavio Forte

flavioforte@hotmail.com

Department of Urology, M.G. Vannini Hospital

Via di Acqua Bullicante 4 - 00177 - Rome (Italy)

Pietro Grande

grandepietro@gmail.com

Sorbonne Université, Assistance Publique-Hôpitaux de Paris, Pitié Salpétière,

Urology Department, Paris (France)

Valerio Olivieri

valerio.olivieri@uniromal.it

Department of Urology, Ivrea Hospital, ASL TO 4, Ivrea (Italy)

Michele Innocenzi

innocenzi.michele@gmail.com

Department of Urology, Bambin Gesù Hospital, Rome (Italy) 